

\section{DISCLAMMER}

Portions of this document may be illegible in electronic image products. Images are produced from the best available original document. 


\title{
One Effect of a Maximum Possible Current Strength
}

\author{
Huang, M. and Heller, L. \\ Biophysics Group, Los Alamos National Laboratory, Los Alamos, NM, USA
}

\section{Introduction}

One of the difficulties encountered when trying to interpret MEG/EEG experimental data is that the spatial extent of the neuronal (primary) current sources is often not known. For some classes of data it is thought that the sources are fairly well localized (focal); while for others they are expected to be distributed over a significant area or volume. In the former case, if the regions of activity are sufficiently small they can be well approximated by point current dipoles. If these are few in number there may be only one, or just a small set of acceptable solutions for the dipole parameters.

At the other extreme one assumes that current is present everywhere in the source space. Even with anatomical constraints taken into account, however, this leads to a highly underdetermined problem. For example, at 1 $\mathrm{cm}$ resolution on the cortex it would take $\approx 2,000$ dipole moments to completely specify the current, and this is much larger than the number of measurements that can be made. Only by imposing physiological constraints can one hope to make this distributed source approach be of any use as a way to determine the actual current. This study examines the extent to which the ambiguity of a distributed source solution is reduced by imposing one specific constraint: A maximum possible value for the dipole moment per unit volume, or per unit area of cortex. There is experimental evidence in support of this limit [1].

We look at the consequences of such a limit on one particular issue, which is of considerable importance in some applications. Given a set of MEG data, can one be certain whether there is, or is not, a significant amount of current in a specified region of the source space? To study this question we choose a simple source space (shown in Fig.1) and subdivide it into two regions, I and II. If a set of magnetic field data can be fit in two different ways: (i) All the current is in region I; (ii) None of the current is in region I; then one cannot be certain whether or not region I contains current.

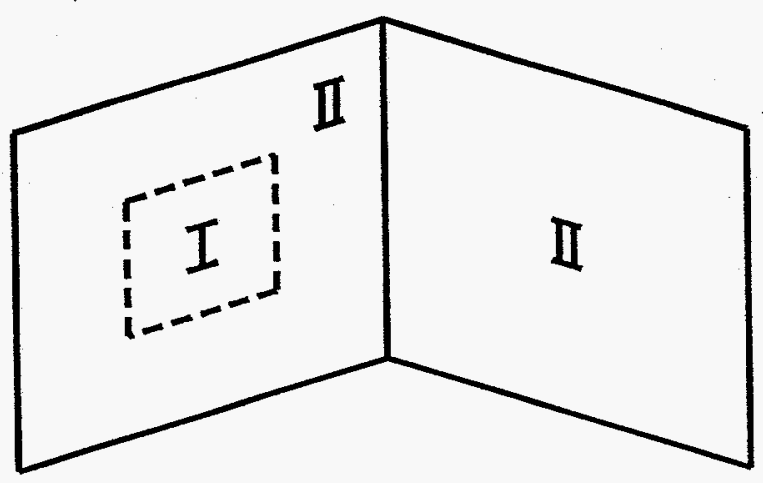

Fig.1. A source space consisting of two perpendicular planes. $I$ is the region being tested. II is the rest of the source space. 
If there are no constraints on the current, this situation can easily arise whenever the number of source parameters (dipole moments) is large compared to the number of measurements. Here we examine the extent to which a constraint on the maximum possible dipole moment per unit area can reduce this ambiguity.

To see why this might happen: Suppose there really is current in region I; a particular reconstruction algorithm may miss that current but still fit the data, by assigning extra current in region II. In this case, a constraint on the maximum possible dipole moment per unit area may be able to prevent this from happening, especially if region I is large.

\section{Source Space and Sensor Array}

To take a first look at this question, we have chosen the simple source space and sensor array shown in Fig.2.

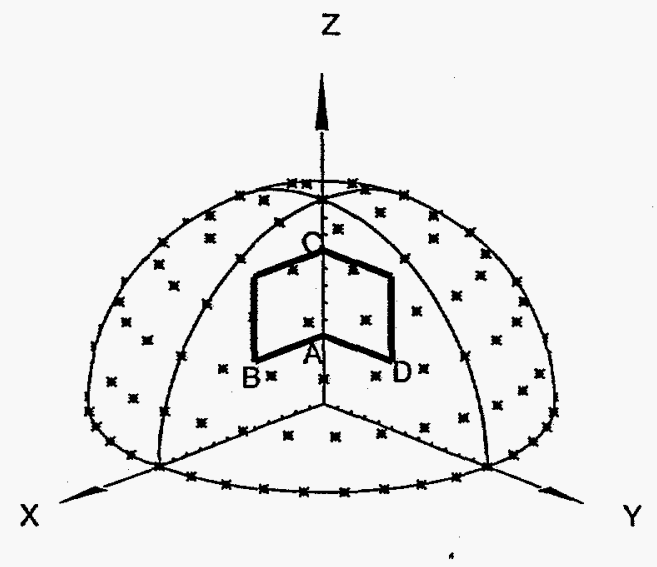

Fig.2. The two planes of the source space, $A B C$ and $A C D$, and the array of magnetic field sensors.

The source space contains portions of two perpendicular planes, $\mathrm{ABC}$ and $\mathrm{ACD}$. Each one is $5 \mathrm{~cm} \times 5 \mathrm{~cm}$ and divided into $28 \times 28$ pixels. The dipoles are normal to the planes (tangential). The total number of dipole moments for this source space is $2 \times 28 \times 28=1,568$. A spherical head model is assumed in our calculation. The sensor array forms a hemisphere $12 \mathrm{~cm}$ from the origin, and 127 radially oriented magnetic field sensors have been assigned to the array.

The number of parameters available to describe a current source $(1,568)$ is very much larger than the number of measurements (127); consequently any given data set can be explained with a very large number $(1,568-127=$ 1,441) of different currents. This leads to a large degree of ambiguity for the inverse problem.

\section{Procedure}

We place a source in the center of the $A B C$ plane as shown in Fig.3. The magnetic field produced by this source is then calculated at all the sensors. There is no noise added to the data. Note that the dipole moment per unit area of this source does not exceed 1 per pixel area. We take this to be the maximum allowed value for the dipole moment per unit area.

Here is the central question: Given this simulated magnetic field data, can we be certain that there is current in the central region of the $\mathrm{ABC}$ plane, if the only constraint is a maximum allowed value for the dipole moment per unit area? To answer this question, we perform the following steps. Force the current to be zero in the 

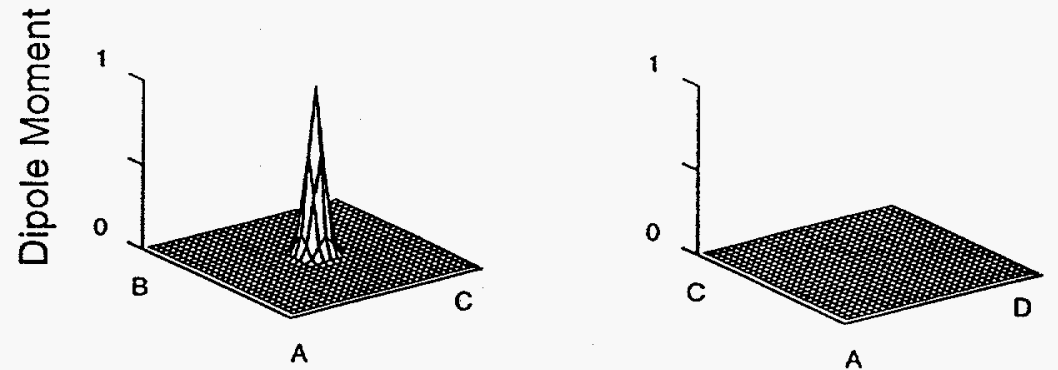

Fig.3. The original current source. It is located in the center of the $\mathrm{ABC}$ plane, has a Gaussian form with standard deviation of one pixel size $(0.18 \mathrm{~cm})$, and a peak dipole moment of 1 (arbitrary units).

(a)
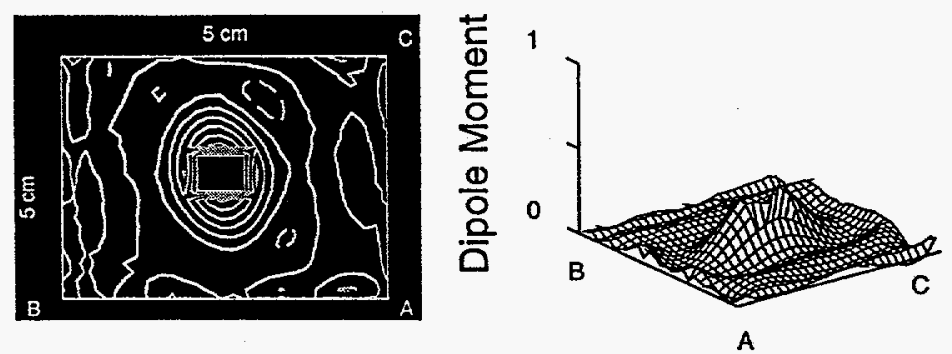

(b)
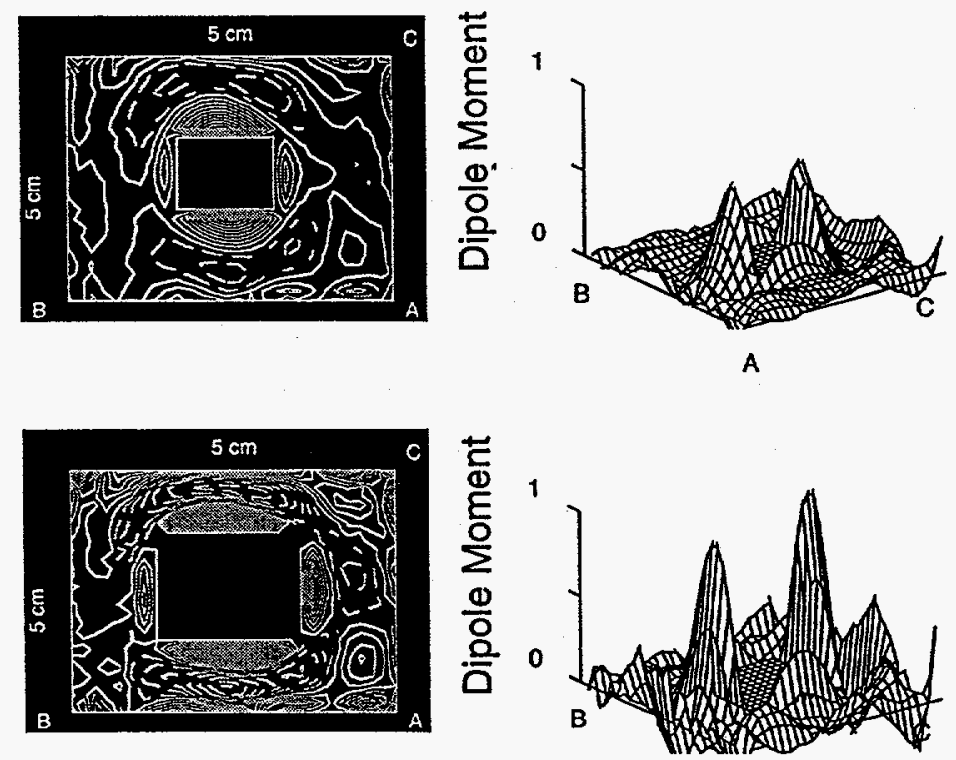

A
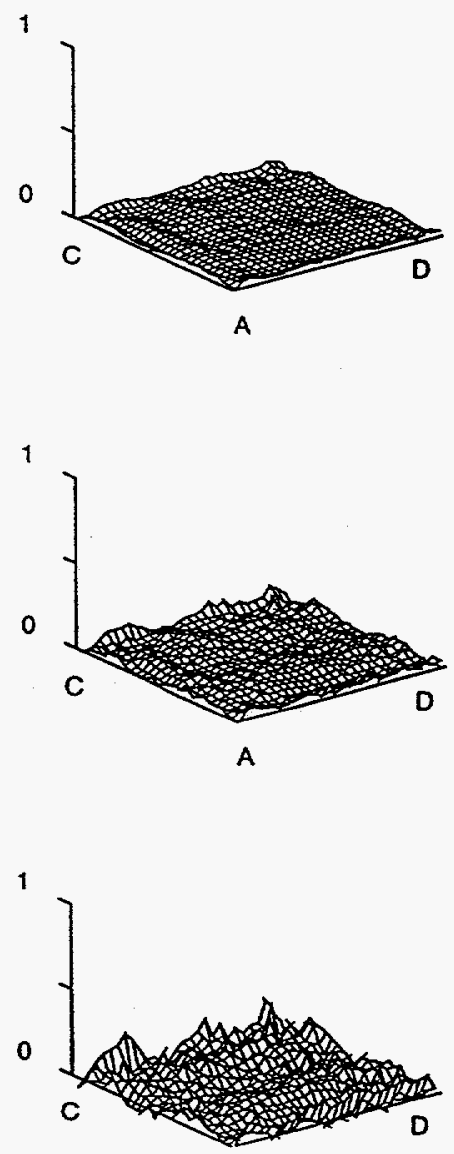

A

Fig.4. Three distributed current sources that produce exactly the same magnetic field (at 127 sensors) as the focal source shown in Fig.3. These sources have zero current in the central region of the $A B C$ plane of size $N \times N$ pixels (pixel size $=0.18 \mathrm{~cm}$ ).

(a) $\mathrm{N}=5(0.9 \mathrm{~cm} \times 0.9 \mathrm{~cm})$; (b) $\mathrm{N}=9(1.6 \mathrm{~cm} \times 1.6 \mathrm{~cm})$; (c) $\mathrm{N}=13(2.3 \mathrm{~cm} \times 2.3 \mathrm{~cm})$.

Left column: contour plot of the dipole moment strength in the $\mathrm{ABC}$ plane.

Center column: 3-D view of the same thing.

Right column: 3-D view of the dipole moment strength in the ACD plane. 
central region of the $A B C$ plane, of size $N \times N$ pixels. Then, construct a distributed current in the remainder of the source space that fits the magnetic field data. Finally, ascertain whether the dipole moment per unit area is everywhere less than the maximum allowed value.

\section{Discussion of Figures 3 and 4}

The current in Fig. 3 is located entirely in a small region in the center of the $\mathrm{ABC}$ plane. The three sources in Fig. 4 have no current whatsoever in the central region of the ABC plane. This region gets larger going from 4 (a) to 4(c). All four sources produce exactly the same magnetic field at all the 127 sensors of the array.

In Fig. 4(a) and 4(b), the dipole moment per unit area is everywhere less than the maximum (1 per pixel area). But in Fig. 4(c), it exceeds the maximum. We find that in an area as large as $2.0 \mathrm{~cm} \times 2.0 \mathrm{~cm}$ one could place all the current, or none of the current, and still fit the data and satisfy the constraint on the maximum dipole moment per unit area.

\section{Conclusion}

If the only constraint that is placed on the allowed current source is anatomical, the ambiguity of the MEG/EEG inverse problem for a distributed source is very great. Indeed, one cannot even know with certainty whether or not a large portion of the source space contains any current! A physiological constraint on the maximum allowed value of the dipole moment per unit area reduces the extent of the ambiguity, but it is still quite large. Additional physiological constraints are needed if distributed source solutions, covering a large area, are to be useful in the electromagnetic inverse problem.

\section{Reference}

[1] Okada Y.C., Papuashvili N., Xu C., Maximum Current Dipole Moment Density as an Important Physiological Constraint in MEG inverse solutions, Proceedings of the Biomag ' 96 Santa Fe Conference.

\section{DISCLAIMER}

This report was prepared as an account of work sponsored by an agency of the United States This report was prepared as an account of work states Government nor any agency thereof, nor any of their Government. Neither the United States Government nor any agency there liability or responsiemployees, makes any warranty, express or implied, or assumes anyation, apparatus, product, or bility for the accuracy, completeness, or usefulness of any information, apparatus, product Referprocess disclosed, or represents that its use would not infringe privately owned re trademark,
ence herein to any specific commercial product, process, or service by trade name, tradecommanufacturer, or otherwise does not necessarily constitute or imply its endorsement, recommendation, or favoring by the United States Government or any agency thereof. The views and opinions of authors expressed herein do not necessarily state or reflect those of the United States Government or any agency thereof. 